\title{
Existence and Exact Asymptotic Behavior of Positive Solutions for a Fractional Boundary Value Problem
}

\author{
Habib Mâagli, Noureddine Mhadhebi, and Noureddine Zeddini \\ King Abdulaziz University, Rabigh Campus, College of Sciences and Arts, Department of Mathematics, P.O. Box 344, \\ Rabigh 21911, Saudi Arabia
}

Correspondence should be addressed to Noureddine Zeddini; noureddine.zeddini@ipein.rnu.tn

Received 4 November 2012; Accepted 25 December 2012

Academic Editor: Chuanzhi Bai

Copyright (C) 2013 Habib Mâagli et al. This is an open access article distributed under the Creative Commons Attribution License, which permits unrestricted use, distribution, and reproduction in any medium, provided the original work is properly cited.

We establish the existence and uniqueness of a positive solution $u$ for the fractional boundary value problem $D^{\alpha} u(x)=-a(x) u^{\sigma}(x)$, $x \in(0,1)$ with the condition $\lim _{x \rightarrow 0} D^{\alpha-1} u(x)=0, u(1)=0$, where $1<\alpha \leq 2, \sigma \in(-1,1)$, and $a$ is a nonnegative continuous function on $(0,1)$ that may be singular at $x=0$ or $x=1$.

\section{Introduction}

Fractional differential equations arise in various fields of science and engineering such as control, porous media, electrochemistry, viscoelasticity, and electromagnetism. They also serve as an excellent tool for the description of hereditary properties of various materials and processes (see[1-3]). In consequence, the subject of fractional differential equations is gaining much importance and attention. Motivated by the surge in the development of this subject, we consider the following problem:

$$
\begin{gathered}
D^{\alpha} u(x)=-a(x) u^{\sigma}(x), \quad x \in(0,1), \\
\lim _{x \rightarrow 0^{+}} D^{\alpha-1} u(x)=0, \quad u(1)=0,
\end{gathered}
$$

where $1<\alpha \leq 2,-1<\sigma<1, a$ is a nonnegative continuous function on $(0,1)$ that may be singular at $x=0$ or $x=1$ and $D^{\alpha}$ is the Riemann-Liouville fractional derivative. Then we study the existence and exact asymptotic behavior of positive solutions for this problem.

We recall that for a measurable function $v$, the RiemannLiouville fractional integral $I_{\beta} v$ and the Riemann-Liouville derivative $D^{\beta} v$ of order $\beta>0$ are, respectively, defined by

$$
\begin{gathered}
I_{\beta} v(x)=\frac{1}{\Gamma(\beta)} \int_{0}^{x}(x-t)^{\beta-1} v(t) d t, \\
D^{\beta} v(x)=\frac{1}{\Gamma(n-\beta)}\left(\frac{d}{d x}\right)^{n} \int_{0}^{x}(x-t)^{n-\beta-1} v(t) d t \\
=\left(\frac{d}{d x}\right)^{n} I_{n-\beta} v(x),
\end{gathered}
$$

provided that the right-hand sides are pointwise defined on $(0,1]$. Here $n=[\beta]+1$ and $[\beta]$ means the integral part of the number $\beta$ and $\Gamma$ is the Euler Gamma function.

Moreover, we have the following well-known properties (see $[2,4])$ :

(i) $I_{\beta} I_{\gamma} v(x)=I_{\beta+\gamma} v(x)$ for $x \in[0,1], v \in L^{1}((0,1]), \beta+$ $\gamma \geq 1$,

(ii) $D^{\beta} I_{\beta} v(x)=v(x)$ for a.e. $x \in[0,1]$, where $v \in$ $L^{1}((0,1]), \beta>0$,

(iii) $D^{\beta} v(x)=0$ if and only if $v(x)=\sum_{j=1}^{n} c_{j} t^{\beta-j}$, where $n=[\beta]+1$ and $\left(c_{1}, c_{2}, \ldots, c_{n}\right) \in \mathbb{R}^{n}$.

Several results are obtained for fractional differential equation with different boundary conditions (see [5-15] and the 
references therein), but none of them deals with the existence of a positive solution for problem (1).

Our aim in this paper is to establish the existence and uniqueness of a positive solution $u \in C_{2-\alpha}([0,1])$ for problem (1) with a precise asymptotic behavior, where $C_{2-\alpha}([0,1])$ is the set of all functions $f$ such that $t \rightarrow t^{2-\alpha} f(t)$ is continuous on $[0,1]$. Note that for $0<\alpha<2$, the solution $u$ for problem (1) blows up at $x=0$.

To state our result, we need some notations. We will use $\mathscr{K}$ to denote the set of Karamata functions $L$ defined on $(0, \eta]$ by

$$
L(t):=c \exp \left(\int_{t}^{\eta} \frac{z(s)}{s} d s\right),
$$

for some $\eta>1$, where $c>0$ and $z \in C([0, \eta])$ such that $z(0)=0$. It is clear that a function $L$ is in $\mathscr{K}$ if and only if $L$ is a positive function in $C^{1}((0, \eta])$ such that

$$
\lim _{t \rightarrow 0^{+}} \frac{t L^{\prime}(t)}{L(t)}=0 .
$$

For two nonnegative functions $f$ and $g$ defined on a set $S$, the notation $f(x) \approx g(x), x \in S$, means that there exists $c>0$ such that $(1 / c) f(x) \leq g(x) \leq c f(x)$, for all $x \in S$. We denote also $x^{+}=\max (x, 0)$ for $x \in \mathbb{R}$.

Throughout this paper we assume that $a$ is nonnegative on $(0,1)$ and satisfies the following condition.

$\left(H_{0}\right) a \in C((0,1))$ such that

$$
a(t) \approx t^{-\lambda} L_{1}(t)(1-t)^{-\mu} L_{2}(1-t), \quad t \in(0,1),
$$

where $\lambda+(2-\alpha) \sigma \leq 1, \mu \leq \alpha, L_{1}, L_{2} \in \mathscr{K}$ satisfying

$$
\int_{0}^{\eta} \frac{L_{1}(t)}{t^{\lambda+(2-\alpha) \sigma}} d t<\infty, \quad \int_{0}^{\eta} \frac{L_{2}(t)}{t^{\mu-\alpha+1}} d t<\infty .
$$

In the sequel, we introduce the function $\theta$ defined on $(0,1)$ by

$$
\theta(x)=\left\{\begin{array}{cc}
1-x \quad & \text { if } \mu<\sigma+\alpha-1, \\
(1-x)\left(\int_{1-x}^{\eta} \frac{L_{2}(s)}{s} d s\right)^{1 /(1-\sigma)} & \text { if } \mu=\sigma+\alpha-1, \\
(1-x)^{(\alpha-\mu) /(1-\sigma)}\left(L_{2}(1-x)\right)^{1 /(1-\sigma)} & \text { if } \sigma+\alpha-1<\mu<\alpha, \\
\left(\int_{0}^{1-x} \frac{L_{2}(s)}{s} d s\right)^{1 /(1-\sigma)} & \text { if } \mu=\alpha .
\end{array}\right.
$$

Our main result is the following.

Theorem 1. Let $\sigma \in(-1,1)$ and assume that a satisfies $\left(H_{0}\right)$. Then problem (1) has a unique positive solution $u \in$ $C_{2-\alpha}([0,1])$ satisfying for $x \in(0,1)$

$$
u(x) \approx x^{\alpha-2} \theta(x) \text {. }
$$

This paper is organized as follows. Some preliminary lemmas are stated and proved in the next section, involving some already known results on Karamata functions. In Section 3, we give the proof of Theorem 1 .

\section{Technical Lemmas}

To keep the paper self-contained, we begin this section by recapitulating some properties of Karamata regular variation theory. The following is due to $[16,17]$.

Lemma 2. The following hold.

(i) Let $L \in \mathscr{K}$ and $\varepsilon>0$, then one has

$$
\lim _{t \rightarrow 0^{+}} t^{\varepsilon} L(t)=0
$$

(ii) Let $L_{1}, L_{2} \in \mathscr{K}$ and $p \in \mathbb{R}$. Then one has $L_{1}+L_{2} \in \mathscr{K}$, $L_{1} L_{2} \in \mathscr{K}$, and $L_{1}^{p} \in \mathscr{K}$.

Example 3. Let $m \in \mathbb{N}^{*}$. Let $c>0,\left(\mu_{1}, \mu_{2}, \ldots, \mu_{m}\right) \in \mathbb{R}^{m}$ and let $d$ be a sufficiently large positive real number such that the function

$$
L(t)=c \prod_{k=1}^{m}\left(\log _{k}\left(\frac{d}{t}\right)\right)^{-\mu_{k}}
$$

is defined and positive on $(0, \eta]$, for some $\eta>1$, where $\log _{k} x=\log \circ \log \circ \cdots \circ \log x$ ( $k$ times). Then $L \in \mathscr{K}$.

Applying Karamata's theorem (see $[16,17])$, we get the following.

Lemma 4. Let $\mu \in \mathbb{R}$ and let $L$ be a function in $\mathscr{K}$ defined on $(0, \eta]$. One has the following.

(i) If $\mu<-1$, then $\int_{0}^{\eta} s^{\mu} L(s) d s$ diverges and $\int_{t}^{\eta} s^{\mu} L(s) d s \sim_{t \rightarrow 0^{+}}-\left(t^{1+\mu} L(t) /(\mu+1)\right)$.

(ii) If $\mu>-1$, then $\int_{0}^{\eta} s^{\mu} L(s) d s$ converges and $\int_{0}^{t} s^{\mu} L(s) d s \sim_{t \rightarrow 0^{+}}\left(t^{1+\mu} L(t) /(\mu+1)\right)$.

Lemma 5. Let $L \in \mathscr{K}$ be defined on $(0, \eta]$. Then one has

$$
\lim _{t \rightarrow 0^{+}} \frac{L(t)}{\int_{t}^{\eta}(L(s) / s) d s}=0 .
$$

If further $\int_{0}^{\eta}(L(s) / s) d s$ converges, then one has

$$
\lim _{t \rightarrow 0^{+}} \frac{L(t)}{\int_{0}^{t}(L(s) / s) d s}=0 .
$$

Proof. We distinguish two cases.

Case 1. We suppose that $\int_{0}^{\eta}(L(s) / s) d s$ converges. Since the function $t \rightarrow L(t) / t$ is nonincreasing in $(0, \omega]$, for some $\omega<$ $\eta$, it follows that, for each $t \leq \omega$, we have

$$
L(t) \leq \int_{0}^{t} \frac{L(s)}{s} d s .
$$

It follows that $\lim _{t \rightarrow 0^{+}} L(t)=0$. So we deduce (11).

Now put

$$
\varphi(t)=\frac{L(t)}{t}, \quad \text { for } t \in(0, \eta)
$$


Using that $\lim _{t \rightarrow 0^{+}}\left(t \varphi^{\prime}(t) / \varphi(t)\right)=-1$, we obtain

$$
\int_{0}^{t} \varphi(s) d s \sim_{t \rightarrow 0^{+}}-\int_{0}^{t} s \varphi^{\prime}(s) d s=-t \varphi(t)+\int_{0}^{t} \varphi(s) d s .
$$

This implies that

$$
\int_{0}^{t} \frac{L(s)}{s} d s \sim_{t \rightarrow 0^{+}}-L(t)+\int_{0}^{t} \frac{L(s)}{s} d s .
$$

So (12) holds.

Case 2. We suppose that $\int_{0}^{\eta}(L(s) / s) d s$ diverges. We have, for some $\omega<\eta$,

$$
\int_{t}^{\omega} \varphi(s) d s \sim_{t \rightarrow 0^{+}} t \varphi(t)-\omega \varphi(\omega)+\int_{t}^{\omega} \varphi(s) d s .
$$

This implies that

$$
\int_{t}^{\omega} \frac{L(s)}{s} d s \sim_{t \rightarrow 0^{+}} L(t)-\omega \varphi(\omega)+\int_{t}^{\omega} \frac{L(s)}{s} d s .
$$

This proves (11) and completes the proof.

Remark 6. Let $L \in \mathscr{K}$ defined on $(0, \eta]$; then, using (4) and (11), we deduce that

$$
t \longrightarrow \int_{t}^{\eta} \frac{L(s)}{s} d s \in \mathscr{K}
$$

If further $\int_{0}^{\eta}(L(s) / s) d s$ converges, we have by (11) that

$$
t \longrightarrow \int_{0}^{t} \frac{L(s)}{s} d s \in \mathscr{K}
$$

Lemma 7. Given $1<\alpha \leq 2$ and $f$ is such that the function $t \rightarrow(1-t)^{\alpha-1} f(t)$ is continuous and integrable on $(0,1)$, then the boundary value problem

$$
\begin{aligned}
& D^{\alpha} u(t)=-f(t), \quad t \in(0,1), \\
& \lim _{x \rightarrow 0} D^{\alpha-1} u(x)=0, \quad u(1)=0,
\end{aligned}
$$

has a unique solution given by

$$
u(x)=G_{\alpha} f(x):=\int_{0}^{1} G_{\alpha}(x, t) f(t) d t,
$$

where

$$
G_{\alpha}(x, t)=\frac{1}{\Gamma(\alpha)}\left[x^{\alpha-2}(1-t)^{\alpha-1}-\left((x-t)^{+}\right)^{\alpha-1}\right],
$$

is the Green function for the boundary value problem (21).

Proof. Since $u_{0}=-I_{\alpha} f$ is a solution of the equation $D^{\alpha} u=$ $-f$, then $D^{\alpha}\left(u+I_{\alpha} f\right)=0$. Consequently there exist two constants $c_{1}, c_{2} \in \mathbb{R}$ such that $u(x)+I_{\alpha} f(x)=c_{1} x^{\alpha-1}+c_{2} x^{\alpha-2}$.
Using the fact that $\lim _{x \rightarrow 0} D^{\alpha-1} u(x)=0$ and $u(1)=0$, we obtain $c_{1}=0$ and $c_{2}=I_{\alpha} f(1)$. So

$$
\begin{aligned}
u(x)= & \frac{1}{\Gamma(\alpha)} x^{\alpha-2} \int_{0}^{1}(1-t)^{\alpha-1} f(t) d t \\
& -\frac{1}{\Gamma(\alpha)} \int_{0}^{x}(x-t)^{\alpha-1} f(t) d t \\
= & \int_{0}^{1} G_{\alpha}(x, t) f(t) d t .
\end{aligned}
$$

In the following, we give some estimates on the function $G_{\alpha}$. So, we need the following lemma.

Lemma 8. For $\lambda, \mu \in(0, \infty)$, and $a, t \in[0,1]$, one has

$$
\min \left(1, \frac{\mu}{\lambda}\right)\left(1-a t^{\lambda}\right) \leq 1-a t^{\mu} \leq \max \left(1, \frac{\mu}{\lambda}\right)\left(1-a t^{\lambda}\right) .
$$

Proposition 9. On $(0,1) \times(0,1)$, one has

$$
G_{\alpha}(x, t) \approx x^{\alpha-2}(1-t)^{\alpha-2}(1-\max (x, t)) .
$$

Proof. For $x, t \in(0,1) \times(0,1)$, we have

$$
G_{\alpha}(x, t)=\frac{(1-t)^{\alpha-1} x^{\alpha-2}}{\Gamma(\alpha)}\left[1-x\left(\frac{(x-t)^{+}}{x(1-t)}\right)^{\alpha-1}\right] .
$$

Since $(x-t)^{+} / x(1-t) \in(0,1)$ for $t \in(0,1)$, then, by applying Lemma 8 with $\mu=\alpha-1$ and $\lambda=1$, we obtain

$$
\begin{aligned}
G_{\alpha}(x, t) & \approx x^{\alpha-2}(1-t)^{\alpha-1}\left(1-\frac{(x-t)^{+}}{1-t}\right) \\
& =x^{\alpha-2}(1-t)^{\alpha-2}(1-\max (x, t)),
\end{aligned}
$$

which completes the proof.

In the sequel we put

$$
b(t)=t^{-\beta} L_{3}(t)(1-t)^{-\gamma} L_{4}(1-t),
$$

where $L_{3}, L_{4} \in \mathscr{K}$ and we aim to give some estimates on $x^{2-\alpha} G_{\alpha} b(x)$.

Proposition 10. Assume that $L_{3}, L_{4} \in \mathscr{K}, \beta \leq 1, \gamma \leq \alpha$ with

$$
\int_{0}^{\eta} t^{-\beta} L_{3}(t) d t<\infty, \quad \int_{0}^{\eta} t^{\alpha-1-\gamma} L_{4}(t) d t<\infty .
$$

Then for $x \in(0,1)$,

$$
x^{2-\alpha} G_{\alpha} b(x) \approx \begin{cases}1-x & \text { if } \gamma<\alpha-1, \\ (1-x) \int_{1-x}^{\eta} \frac{L_{4}(t)}{t} d t & \text { if } \gamma=\alpha-1, \\ (1-x)^{\alpha-\gamma} L_{4}(1-x) & \text { if } \alpha-1<\gamma<\alpha, \\ \int_{0}^{1-x} \frac{L_{4}(t)}{t} d t & \text { if } \gamma=\alpha .\end{cases}
$$


Proof. Using Proposition 9, we have

$$
\begin{aligned}
x^{2-\alpha} G_{\alpha} b(x) \approx & (1-x) \int_{0}^{x}(1-t)^{\alpha-2-\gamma} t^{-\beta} L_{3}(t) L_{4}(1-t) d t \\
& +\int_{x}^{1}(1-t)^{\alpha-1-\gamma} t^{-\beta} L_{3}(t) L_{4}(1-t) d t \\
= & (1-x) I(x)+J(x) .
\end{aligned}
$$

For $0<x \leq 1 / 2$, we use Lemma 4 and hypotheses (30) to deduce that

$$
\begin{gathered}
I(x) \approx \begin{cases}x^{1-\beta} L_{3}(x) & \text { if } \beta<1, \\
\int_{0}^{x} \frac{L_{3}(t)}{t} d t & \text { if } \beta=1,\end{cases} \\
J(x) \approx \int_{1 / 2}^{1}(1-t)^{\alpha-1-\gamma} L_{4}(1-t) d t+\int_{x}^{1 / 2} t^{-\beta} L_{3}(t) d t \\
\approx 1+\int_{x}^{1 / 2} t^{-\beta} L_{3}(t) d t \\
\approx 1 .
\end{gathered}
$$

Hence, it follows from Lemma 2 and hypothesis (30) that, for $0<x \leq 1 / 2$, we have

$$
x^{2-\alpha} G_{\alpha} b(x) \approx 1 .
$$

Now, for $1 / 2 \leq x<1$, we use again Lemma 4 and hypothesis (30) to deduce that

$$
\begin{aligned}
I(x) & \approx \int_{0}^{1 / 2} t^{-\beta} L_{3}(t) d t+\int_{1 / 2}^{x}(1-t)^{\alpha-2-\gamma} L_{4}(1-t) d t \\
& \approx 1+\int_{1-x}^{1 / 2} t^{\alpha-2-\gamma} L_{4}(t) d t \\
& \approx \begin{cases}1 & \text { if } \gamma<\alpha-1, \\
\int_{1-x}^{\eta} \frac{L_{4}(t)}{t} d t & \text { if } \gamma=\alpha-1, \\
(1-x)^{\alpha-1-\gamma} L_{4}(1-x) & \text { if } \gamma>\alpha-1,\end{cases} \\
J(x) & \approx \begin{cases}1-x & t^{\alpha-1-\gamma} L_{4}(t) d t\end{cases} \\
& \approx \begin{cases}(1-x)^{\alpha-\gamma} L_{4}(1-x) & \text { if } \gamma<\alpha, \\
\int_{0}^{1-x} \frac{L_{4}(t)}{t} d t & \text { if } \gamma=\alpha .\end{cases}
\end{aligned}
$$

Hence, it follows from Lemmas 2 and 5 that, for $x \in[1 / 2,1)$, we have

$$
x^{2-\alpha} G_{\alpha} b(x) \approx \begin{cases}1-x & \text { if } \gamma<\alpha-1, \\ (1-x) \int_{1-x}^{\eta} \frac{L_{4}(t)}{t} d t & \text { if } \gamma=\alpha-1, \\ (1-x)^{\alpha-\gamma} L_{4}(1-x) & \text { if } \alpha-1<\gamma<\alpha, \\ \int_{0}^{1-x} \frac{L_{4}(t)}{t} d t & \text { if } \gamma=\alpha .\end{cases}
$$

This together with (34) implies that (36) holds on $(0,1)$.

\section{Proof of Theorem 1}

We begin this section by giving a preliminary result that will play a crucial role in the proof of Theorem 1.

Proposition 11. Assume that the function a satisfies $\left(H_{0}\right)$ and put $\omega(t)=a(t) t^{(\alpha-2) \sigma} \theta^{\sigma}(t)$ for $t \in(0,1)$. Then one has, for $x \in(0,1)$,

$$
x^{2-\alpha} G_{\alpha} \omega(x) \approx \theta(x) .
$$

Proof. For $t \in(0,1)$, we have

$$
\begin{aligned}
\omega(t)= & a(t) t^{(\alpha-2) \sigma} \theta^{\sigma}(t) \\
= & \left\{\begin{array}{c}
t^{-\lambda-(2-\alpha) \sigma}(1-t)^{-\mu+\sigma} L_{1}(t) L_{2}(1-t) \\
\text { if } \mu<\sigma+\alpha-1, \\
t^{-\lambda-(2-\alpha) \sigma}(1-t)^{-\mu+\sigma} L_{1}(t) L_{2}(1-t) \\
\quad \times\left(\int_{1-t}^{\eta} \frac{L_{2}(s)}{s} d s\right)^{\sigma /(1-\sigma)} \quad \text { if } \mu=\sigma+\alpha-1, \\
t^{-\lambda-(2-\alpha) \sigma}(1-t)^{-(\mu-\sigma \alpha) /(1-\sigma)} L_{1}(t) \\
\times\left(L_{2}(1-t)\right)^{\sigma /(1-\sigma)} \text { if } \sigma+\alpha-1<\mu<\alpha, \\
t^{-\lambda-(2-\alpha) \sigma}(1-t)^{-\mu} L_{1}(t) L_{2}(1-t) \\
\times\left(\int_{0}^{1-t} \frac{L_{2}(s)}{s} d s\right)^{\sigma /(1-\sigma)} \quad \text { if } \mu=\alpha .
\end{array}\right.
\end{aligned}
$$

So, we can see that

$$
\omega(t)=t^{-\beta}(1-t)^{-\gamma} \widetilde{L}_{1}(t) \widetilde{L}_{2}(1-t),
$$

where $\beta \leq 1, \gamma \leq \alpha$ and, according to Lemma 2 , the functions $t \rightarrow \widetilde{L}_{1}(t)$ and $t \rightarrow \widetilde{L}_{2}(t)$ are in $\mathscr{K}$. Moreover, using Lemma 4 , we have $\int_{0}^{\eta} t^{-\beta} \widetilde{L}_{1}(t) d t<\infty$ and $\int_{0}^{\eta} t^{-\gamma} \widetilde{L}_{2}(t) d t<$ $\infty$. So the result follows from Proposition 10.

Proof of Theorem 1. From Proposition 11, there exists $M>1$ such that for each $x \in(0,1)$

$$
\frac{1}{M} \theta(x) \leq x^{2-\alpha} G_{\alpha} \omega(x) \leq M \theta(x)
$$

where $\omega(t)=a(t) t^{(\alpha-2) \sigma} \theta^{\sigma}(t)$.

Put $c_{0}=M^{1 /(1-|\sigma|)}$ and let

$$
\Lambda=\left\{v \in C([0,1]): \frac{1}{c_{0}} \theta \leq v \leq c_{0} \theta\right\} .
$$

In order to use a fixed point theorem, we denote $\widetilde{a}(t)=$ $a(t) t^{(\alpha-2) \sigma}$ and we define the operator $T$ on $\Lambda$ by

$$
T v(x)=x^{2-\alpha} G_{\alpha}\left(\tilde{a} v^{\sigma}\right)(x) .
$$

For this choice of $c_{0}$, we can easily prove that for $v \in \Lambda$, we have $T v \leq c_{0} \theta$ and $T v \geq\left(1 / c_{0}\right) \theta$. 
Now, we have

$$
\begin{gathered}
T v(x)=\frac{x^{2-\alpha}}{\Gamma(\alpha)} \int_{0}^{1} G_{\alpha}(x, t) \tilde{a}(t) v^{\sigma}(t) d t \\
=\frac{1}{\Gamma(\alpha)} \int_{0}^{1}\left[(1-t)^{\alpha-1}-x^{2-\alpha}\left((x-t)^{+}\right)^{\alpha-1}\right] \\
\times \tilde{a}(t) v^{\sigma}(t) d t .
\end{gathered}
$$

Since the function $(x, t) \rightarrow(1-t)^{\alpha-1}-x^{2-\alpha}\left((x-t)^{+}\right)^{\alpha-1}$ is continuous on $[0,1] \times[0,1]$ and the function $t \rightarrow(1-$ $t)^{\alpha-1} \widetilde{a}(t) \theta^{\sigma}(t)$ is integrable on $(0,1)$, we deduce that the operator $T$ is compact from $\Lambda$ to itself. It follows by the Schauder fixed point theorem that there exists $v \in \Lambda$ such that $T v=v$. Put $u(x)=x^{\alpha-2} v(x)$. Then $u \in C_{2-\alpha}([0,1])$ and $u$ satisfies the equation

$$
u(x)=G_{\alpha}\left(a u^{\sigma}\right)(x)
$$

Since the function $t \rightarrow(1-t)^{\alpha-1} a(t) u^{\sigma}(t)$ is continuous and integrable on $(0,1)$, then by Lemma 7 the function $u$ is a positive continuous solution of problem (1).

Finally, let us prove that $u$ is the unique positive continuous solution satisfying (8). To this aim, we assume that (1) has two positive solutions $u, v \in C_{2-\alpha}([0,1])$ satisfying (8) and consider the nonempty set $J=\{m \geq 1: 1 / m \leq u / v \leq m\}$ and put $c=\inf J$. Then $c \geq 1$ and we have $(1 / c) v \leq u \leq c v$. It follows that $u^{\sigma} \leq c^{|\sigma|} v^{\sigma}$ and consequently

$$
\begin{gathered}
-D^{\alpha}\left(c^{|\sigma|} v-u\right)=a\left(c^{|\sigma|} v^{\sigma}-u^{\sigma}\right) \geq 0, \\
\lim _{t \rightarrow 0^{+}} D^{\alpha-1}\left(c^{|\sigma|} v-u\right)(t)=0, \quad\left(c^{|\sigma|} v-u\right)(1)=0 .
\end{gathered}
$$

Which implies by Lemma 7 that $c^{|\sigma|} v-u=G_{\alpha}\left(a\left(c^{|\sigma|} v^{\sigma}-\right.\right.$ $\left.\left.u^{\sigma}\right)\right) \geq 0$. By symmetry, we obtain also that $v \leq c^{|\sigma|} u$. Hence $c^{|\sigma|} \in J$ and $c \leq c^{|\sigma|}$. Since $|\sigma|<1$, then $c=1$ and consequently $u=v$.

Example 12. Let $\sigma \in(-1,1)$ and $a$ be a positive continuous function on $(0,1)$ such that

$$
a(t) \approx(1-t)^{-\mu} \log \left(\frac{3}{1-t}\right)^{-\beta}
$$

where $\mu<\alpha$ and $\beta \in \mathbb{R}$ or $\mu=\alpha$ and $\beta>1$. Then, using Theorem 1, problem (1) has a unique positive continuous solution $u$ satisfying the following estimates.

(i) If $\mu<\sigma+\alpha-1$ or $\mu=\sigma+\alpha-1$ and $\beta>1$, then for $x \in(0,1)$,

$$
u(x) \approx x^{\alpha-2}(1-x)
$$

(ii) If $\mu=\sigma+\alpha-1$ and $\beta=1$, then for $x \in(0,1)$,

$$
u(x) \approx x^{\alpha-2}(1-x)\left[\log \left(\log \left(\frac{3}{1-x}\right)\right)\right]^{1 /(1-\sigma)}
$$

(iii) If $\mu=\sigma+\alpha-1$ and $\beta<1$, then for $x \in(0,1)$,

$$
u(x) \approx x^{\alpha-2}(1-x)\left[\log \left(\frac{3}{1-x}\right)\right]^{(1-\beta) /(1-\sigma)} .
$$

(iv) If $\sigma+\alpha-1<\mu<\alpha$, then for $x \in(0,1)$,

$u(x) \approx x^{\alpha-2}(1-x)^{(\alpha-\mu) /(1-\sigma)}\left[\log \left(\frac{3}{1-x}\right)\right]^{-\beta /(1-\sigma)}$.

(v) If $\mu=\alpha$ and $\beta>1$, then for $x \in(0,1)$,

$$
u(x) \approx x^{\alpha-2}\left[\log \left(\frac{3}{1-x}\right)\right]^{(1-\beta) /(1-\sigma)} .
$$

\section{Acknowledgment}

The authors thank the anonymous referees for a careful reading of the paper and for their helpful suggestions.

\section{References}

[1] K. Diethelm and A. D. Freed, "On the solution of nonlinear fractional order differential equations used in the modelling of viscoplasticity," in Scientific Computing in Chemical Engineering II-Computational Fluid Dynamics, Reaction Engineering and Molecular Properties, F. Keil, W. Mackens, and H. Voss, Eds., pp. 217-224, Springer, Heidelberg, Germany, 1999.

[2] A. A. Kilbas, H. M. Srivastava, and J. J. Trujillo, Theory and Applications of Fractional Differential Equations, Elsevier, Amsterdam, The Netherlands, 2006.

[3] W. Lin, "Global existence theory and chaos control of fractional differential equations," Journal of Mathematical Analysis and Applications, vol. 332, no. 1, pp. 709-726, 2007.

[4] I. Podlubny, Fractional Differential Equations, vol. 198 of Mathematics in Science and Engineering, Academic Press, San Diego, Calif, USA, 1999.

[5] R. P. Agarwal, D. O’Regan, and S. Staněk, "Positive solutions for Dirichlet problems of singular nonlinear fractional differential equations," Journal of Mathematical Analysis and Applications, vol. 371, no. 1, pp. 57-68, 2010.

[6] R. P. Agarwal, M. Benchohra, S. Hamani, and S. Pinelas, "Boundary value problems for differential equations involving Riemann-Liouville fractional derivative on the half-line," Dynamics of Continuous, Discrete \& Impulsive Systems A, vol. 18, no. 2, pp. 235-244, 2011.

[7] Z. Bai and H. Lü, "Positive solutions for boundary value problem of nonlinear fractional differential equation," Journal of Mathematical Analysis and Applications, vol. 311, no. 2, pp. 495-505, 2005.

[8] B. Ahmad, "Existence of solutions for irregular boundary value problems of nonlinear fractional differential equations," Applied Mathematics Letters, vol. 23, no. 4, pp. 390-394, 2010.

[9] B. Ahmad and J. J. Nieto, "Riemann-Liouville fractional integrodifferential equations with fractional nonlocal integral boundary conditions," Boundary Value Problems, vol. 2011, article 36, 2011.

[10] J. Caballero, J. Harjani, and K. Sadarangani, "Positive solutions for a class of singular fractional boundary value problems," Computers \& Mathematics with Applications, vol. 62, no. 3, pp. 1325-1332, 2011. 
[11] J. Deng and L. Ma, "Existence and uniqueness of solutions of initial value problems for nonlinear fractional differential equations," Applied Mathematics Letters, vol. 23, no. 6, pp. 676680, 2010.

[12] N. Kosmatov, "A singular boundary value problem for nonlinear differential equations of fractional order," Journal of Applied Mathematics and Computing, vol. 29, no. 1-2, pp. 125-135, 2009.

[13] Y. Liu, W. Zhang, and X. Liu, "A sufficient condition for the existence of a positive solution for a nonlinear fractional differential equation with the Riemann-Liouville derivative," Applied Mathematics Letters, vol. 25, no. 11, pp. 1986-1992, 2012.

[14] T. Qiu and Z. Bai, "Existence of positive solutions for singular fractional differential equations," Electronic Journal of Differential Equations, vol. 149, 19 pages, 2008.

[15] Y. Zhao, S. Sun, Z. Han, and Q. Li, "Positive solutions to boundary value problems of nonlinear fractional differential equations," Abstract and Applied Analysis, vol. 2011, Article ID 390543, 16 pages, 2011.

[16] V. Marić, Regular Variation and Differential Equations, vol. 1726 of Lecture Notes in Mathematics, Springer, Berlin, Germany, 2000.

[17] E. Seneta, Regularly Varying Functions, vol. 508 of Lecture Notes in Mathematics, Springer, Berlin, Germany, 1976. 


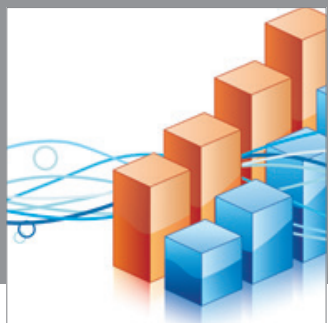

Advances in

Operations Research

mansans

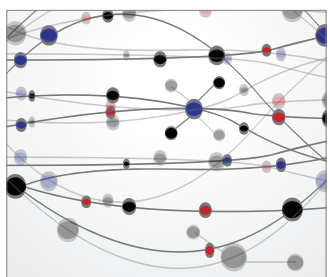

The Scientific World Journal
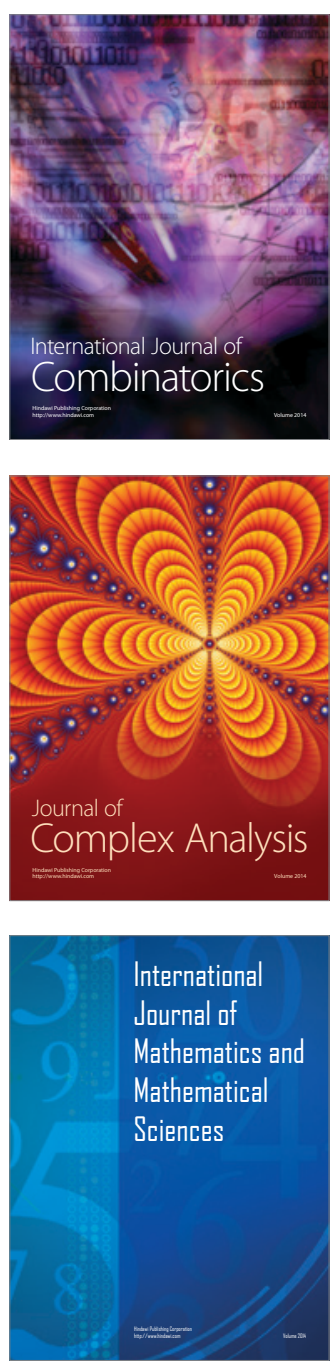
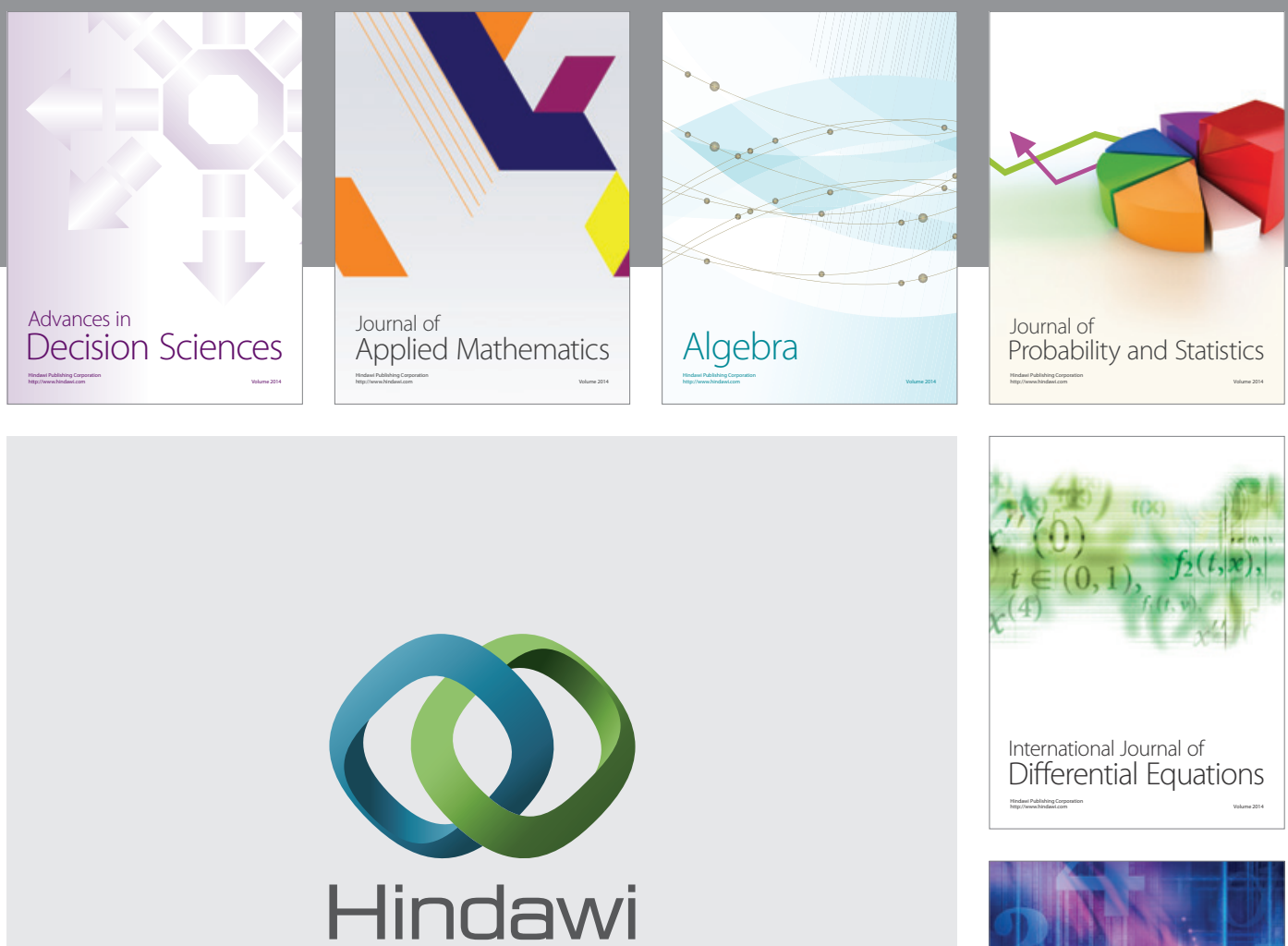

Submit your manuscripts at http://www.hindawi.com
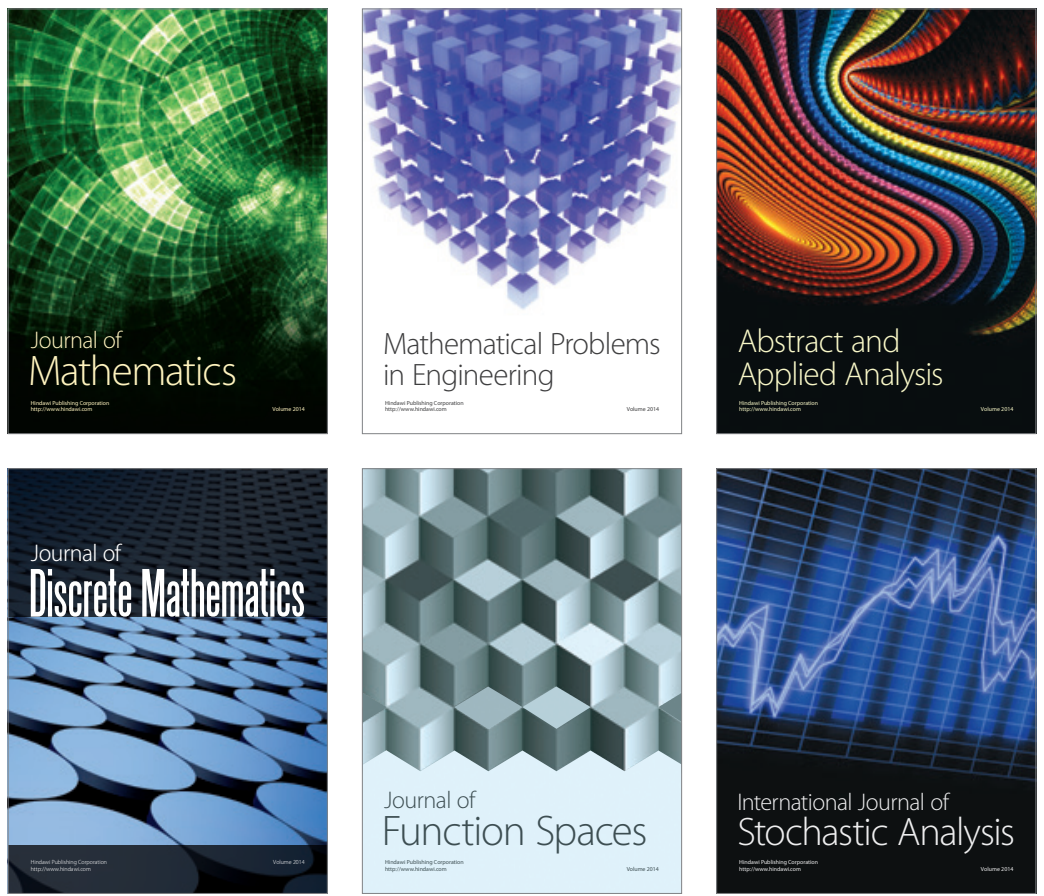

Journal of

Function Spaces

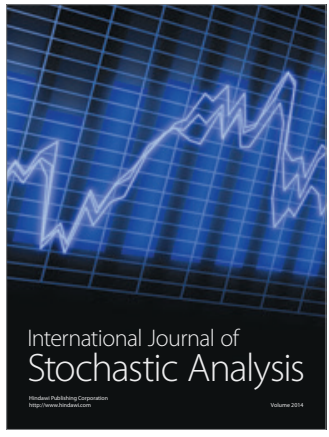

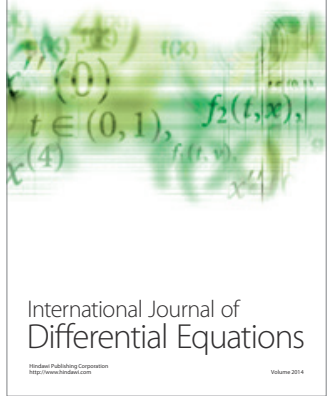
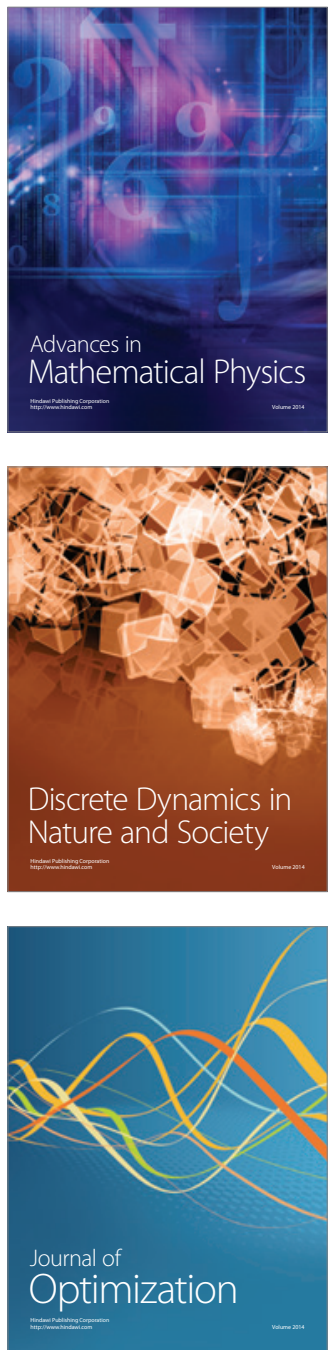